\title{
ARTICLE Progressive brain structural alterations assessed via causal analysis in patients with generalized anxiety disorder
}

\author{
Yuyan Chen ${ }^{1}$, Qian Cui ${ }^{2}$, Yun-Shuang Fan ${ }^{1}$, Xiaonan Guo ${ }^{1}$, Qin Tang ${ }^{1}$, Wei Sheng ${ }^{1}$, Ting Lei ${ }^{2}$, Di Li ${ }^{1}$, Fengmei Lu ${ }^{1}$, Zongling He ${ }^{1}$, \\ Yang Yang ${ }^{1}$, Shan $\mathrm{Hu}^{2}$, Jiaxin Deng ${ }^{2}$ and Huafu Chen ${ }^{1,3,4}$
}

Accumulating neuroimaging studies implicate widespread brain structural alterations in patients with generalized anxiety disorder (GAD), but little is known regarding the temporal information of these changes and their causal relationships. In this study, a morphometric analysis was performed on T1-weighted structural images, and the progressive changes in the gray matter volume (GMV) in GAD were simulated by dividing the patients into different groups from low illness duration to high illness duration. The duration was defined as the interval between the onset of GAD and the time for magnetic resonance imaging collection. Then, a causal structural covariance network analysis was conducted to describe the causal relationships of the brain structural alterations in GAD. With increased illness duration, the GMV reduction in GAD originated from the subgenual anterior cingulate cortex (sgACC) and propagated to the bilateral ventromedial prefrontal cortex, right dorsomedial prefrontal cortex, left inferior temporal gyrus, and right insula. Intriguingly, the sgACC and the right insula had positive causal effects on each other. Moreover, both sgACC and right insula exhibited positive causal effects on the parietal cortex and negative effects on the posterior cingulate cortex, dorsolateral prefrontal cortex, visual cortex, and temporal lobe. The opposite causal effects were noted on the somatosensory and the ventrolateral prefrontal cortices. In conclusion, patients with GAD show gradual GMV reduction with increasing ilness duration. Furthermore, the causal effects of the sgACC and the right insula GMV reduction with shifts of duration may provide an important new avenue for understanding the pathological anomalies in GAD.

Neuropsychopharmacology (2020) 45:1689-1697; https://doi.org/10.1038/s41386-020-0704-1

\section{INTRODUCTION}

Generalized anxiety disorder (GAD) is a chronic, persistent, and disabling mental disorder [1,2] with core symptoms of excessive and uncontrollable worry [3, 4]. Patients with GAD are frequently caught in repetitive negative thinking [5] and disturbed by a series of physical and psychological symptoms, including irritability, feelings of restlessness, difficulty in concentrating, and sleep problems [6]. Widespread brain structural alterations in GAD have been reported in a substantial body of brain imaging research. However, little is known about the relationships of the structural alterations in this disease.

A recent review [7] has summarized the structural alterations of GAD by reviewing the previous literature. A total of 35 studies with several structural analysis methodologies are included in this review, and 16 studies are conducted using voxel-based morphometry (VBM), which may shed light on the popularity of the VBM in investigating the neuroanatomical alterations in GAD. Among various morphological properties characterized by VBM, the gray matter volume (GMV) analysis appears predominant and provides important evidence for understanding the neuroanatomical basis of the cognitive and affective disruptions in GAD [8-10]. The current findings on the GMV alterations in GAD show inconsistencies. According to the previous literature, different parts of the prefrontal cortex [11-15], anterior cingulate cortex (ACC) [13, 14], and precuneus $[12,13]$ exhibit different tendencies of GMV alterations in GAD. Moreover, the primary somatosensory cortex $[12,16]$ and superior temporal gyrus [17-19] display conflicting GMV changes in different studies. This controversy may be driven by the heterogeneity of the experiment designs, patient characteristics, and analytical methodologies. The most consistent findings involve increased GMV in the amygdala $[11,15,16,20]$ and the putamen $[21,22]$ and decreased GMV in the insula, hippocampus, midbrain, and thalamus $[17,18]$ in GAD. Aside from the above differences between patients and healthy controls $(\mathrm{HC})$, some studies have reported the gender differences in GAD. Males with GAD have larger GMV in the precuneus/posterior cingulate cortex (PCC) [22] and smaller GMV in the dorsolateral prefrontal cortex (DLPFC) [17] compared with females with GAD. The existing research on the GMV changes in GAD suggests multiregional structural abnormalities. To the best of our knowledge, no neuroimaging study has investigated the causality between these changes.

Zhang and colleagues proposed a novel method called causal structural covariance network (CaSCN) analysis [23] to characterize the causal relationships of brain structural alterations with increasing illness duration. This method is conducted by applying the signedpath coefficient Granger causality (GC) analysis to T1-weighted

\footnotetext{
${ }^{1}$ The Clinical Hospital of Chengdu Brain Science Institute, School of Life Science and Technology, University of Electronic Science and Technology of China, Chengdu, China; ${ }^{2}$ School of Public Affairs and Administration, University of Electronic Science and Technology of China, Chengdu, China; ${ }^{3}$ MOE Key Lab for Neuroinformation; High-Field Magnetic

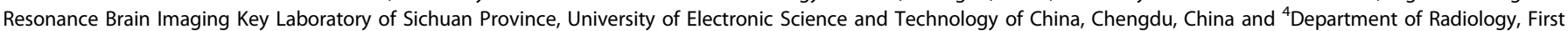
Affiliated Hospital to Army Medical University, Chongqing, China

Correspondence: Qian Cui (qiancui26@gmail.com) or Huafu Chen (chenhf@uestc.edu.cn)
}

Received: 4 December 2019 Revised: 13 April 2020 Accepted: 4 May 2020

Published online: 12 May 2020 
structural images. The GC approach is dependent on time and often applied on functional imaging data, which have a series of time course [24-28]. Thus, before starting the CaSCN computation, Zhang and colleagues have sequenced the structural images of patients with epilepsy in accordance with their illness duration to obtain the time-series information on the gradual alteration property of the brain structure in epilepsy [23]. The GMV reduction in the hippocampus exerts causal effects on numerous brain regions involved in the epileptic network. This method is likewise used to investigate the temporal information and causal relationships of GMV alterations with increasing illness duration of schizophrenia [26] and major depressive disorder (MDD) [29]. The thalamus is the origin of progressive GMV alterations in schizophrenia and exhibits causal influences on other regions, an outcome that has an implication on the early intervention for this disease. Besides, the hippocampus projects causal effects on the amygdala and the default mode network (DMN) regions in MDD, thereby showing the network spread mechanism of structural alterations in MDD. Whether patients with GAD will also show progressive morphological alterations along with increasing illness duration and whether these changes have some causal effects remain unclear.

This study aimed to investigate the GMV changes in GAD as the illness duration increases and explore the causal relationships between these changes. GMV comparative analyses were conducted at different stages of illness duration of GAD to assess whether the morphological changes in GAD exhibit gradual changing patterns. These comparative analyses can help us identify some critical regions, such as the regions in which the structural changes started and with longer duration of GAD. These regions were assumed to be valuable for understanding the occurrence and maintenance of anxiety. Subsequently, the CaSCN analysis based on the critical regions were performed to track the causal relationships of the GMV changes in GAD and the regions influenced by these critical regions. The GMV of patients with GAD was expected to gradually change as the illness duration increased, and some causalities were expected to exist among these changes. Considering the different prevalence and clinical features of males and females with GAD [30-32], the gender effect on the GMV was conducted. These analyses were used as supplemental analyses rather than the main findings, which aimed to make our findings comprehensive and provide some clues for future gender-related studies.

\section{MATERIALS AND METHODS}

\section{Participants}

A total of 75 patients with GAD from the Clinical Hospital of Chengdu Brain Science Institute, University of Electronic Science and Technology of China (UESTC) were initially recruited. Three patients were excluded because of the poor quality of their images. Thus, the final sample size consisted of 72 patients. Two experienced psychiatrists diagnosed patients by using the Structured Clinical Interview for DSM-IV-TR-Patient Edition (SCID$P, 2 / 2001$ revision). All patients met the criteria for GAD. The illness duration was defined as the interval between the onset of GAD and the time for magnetic resonance imaging (MRI) acquisition of each patient. Patients with schizophrenia, personality disorder, substance abuse, neurological illness, or history of loss of consciousness were excluded. The 14-item Hamilton Anxiety Rating Scale (HAMA) was utilized to assess the severity of anxiety of patients. Most patients were prescribed anxiolytic drugs, and a few of them were simultaneously prescribed with antipsychotic medications. Table 1 presents the detailed medical information of patients. A total of 57 age-, gender-, handedness-, and years of education-matched $\mathrm{HC}$ were recruited from the local community through advertisements. The SCID non-patient edition was applied to ensure that the $\mathrm{HC}$ had no history of psychopathologic conditions. This study was listed on Clinical-Trials.gov (Registration Number: NCT02888509) and approved by the research ethical committee of the UESTC. Written informed consents were obtained from all participants after they received a detailed description of the study procedures.

\section{Data acquisition}

The participants were scanned using a 3T GE DISCOVERY MR750 scanner (General Electric, Fairfield Connecticut, USA) with an 8-channel prototype quadrature birdcage head coil. Highresolution structural T1-weighted images were acquired using the following parameters: repetition time/echo time $=5.92 / 1.956 \mathrm{~ms}$, matrix size $=256 \times 256$, flip angle $=12^{\circ}$, field of view $=256 \mathrm{~mm} \times$ $256 \mathrm{~mm}$, voxel size $=1 \mathrm{~mm} \times 1 \mathrm{~mm} \times 1 \mathrm{~mm}$, slices $=156$, slice thickness $=1 \mathrm{~mm}$, and no gap.

\section{Data preprocessing}

The VBM8 toolbox (http://dbm.neuro.uni-jena.de/vbm8) implemented in the SPM8 (http://www.fil.ion.ucl.ac.uk/spm) was employed to preprocess the structural T1-weighted images. First, the artifacts of the images were checked, and the origins of the images were adjusted to the anterior commissure. Subsequently, the images of each participant were normalized to the standard Montreal Neurological Institute template by using an affine followed by nonlinear transformation and resampled to $1.5 \mathrm{~mm}$ $\times 1.5 \mathrm{~mm} \times 1.5 \mathrm{~mm}$. The normalized images were segmented into GMV, white matter volume, and cerebral spinal fluid maps. The data quality of the segmented maps was checked. The probabilistic gray matter (GM) maps were further smoothed using an $8 \mathrm{~mm}$ full width at half maximum Gaussian kernel. The smoothed GM images were subjected to the following analyses.

\section{Medication information-related assessment}

We assessed the medication information of patients by using the total medication load index, an indicator calculated using a widely used method in neuropsychiatric studies [33-38]. Each medication each patient had taken was coded ( 0 , absent; 1 , low dose; 2 , high dose) in accordance with the previous criteria [39]. In detail, each medication of each patient was converted into a 4-level classification with reference to these criteria that closely correlated the dosage and the duration the patient had taken the medicine. Then, the medications on levels 1 and 2 were coded as low dose, and those on levels 3 and 4 were coded as high dose. We added a no-dose subtype for the patients who were not taking any medication. Moreover, escitalopram and duloxetine were two medications that were not included in the criteria proposed by Sackeim [39] and were coded as 0, 1, or 2 in accordance with the midpoint of the daily dose range recommended by the Physician's-Desk-Reference. Finally, all the codes for all medications one patient had taken were added, and this value was applied as the total medication load index of the patient.

\section{Overall GMV alterations in patients with GAD}

A two-sample $t$-test was performed to investigate the overall GMV changes in GAD. The age, gender, years of education, and total intracranial volume (TIV) of the participants were used as covariates. The Gaussian random field (GRF) correction (voxel level $p<0.001$, cluster level $p<0.05$ ) was conducted to test the statistical significance of the difference between the GMV of the two groups. The regions that survived the multiple comparison correction were considered as regions of interest (ROls). The Pearson's correlation analysis of the GMV of each ROI and HAMA scores of GAD was performed to examine the influence of the symptom severity of patients on their morphological changes. The gender, age, years of education, TIV, illness duration, illness onset, total medication load index, number of anxiety episodes, and duration of single anxiety episode of patients were used as regressors. Also, the GMV of each $\mathrm{ROI}$ and the illness onset of GAD were subjected to Spearman correlation analysis, where the covariates were similar with the above variables except the 
Table 1. Characteristics of demographic and clinical variables.

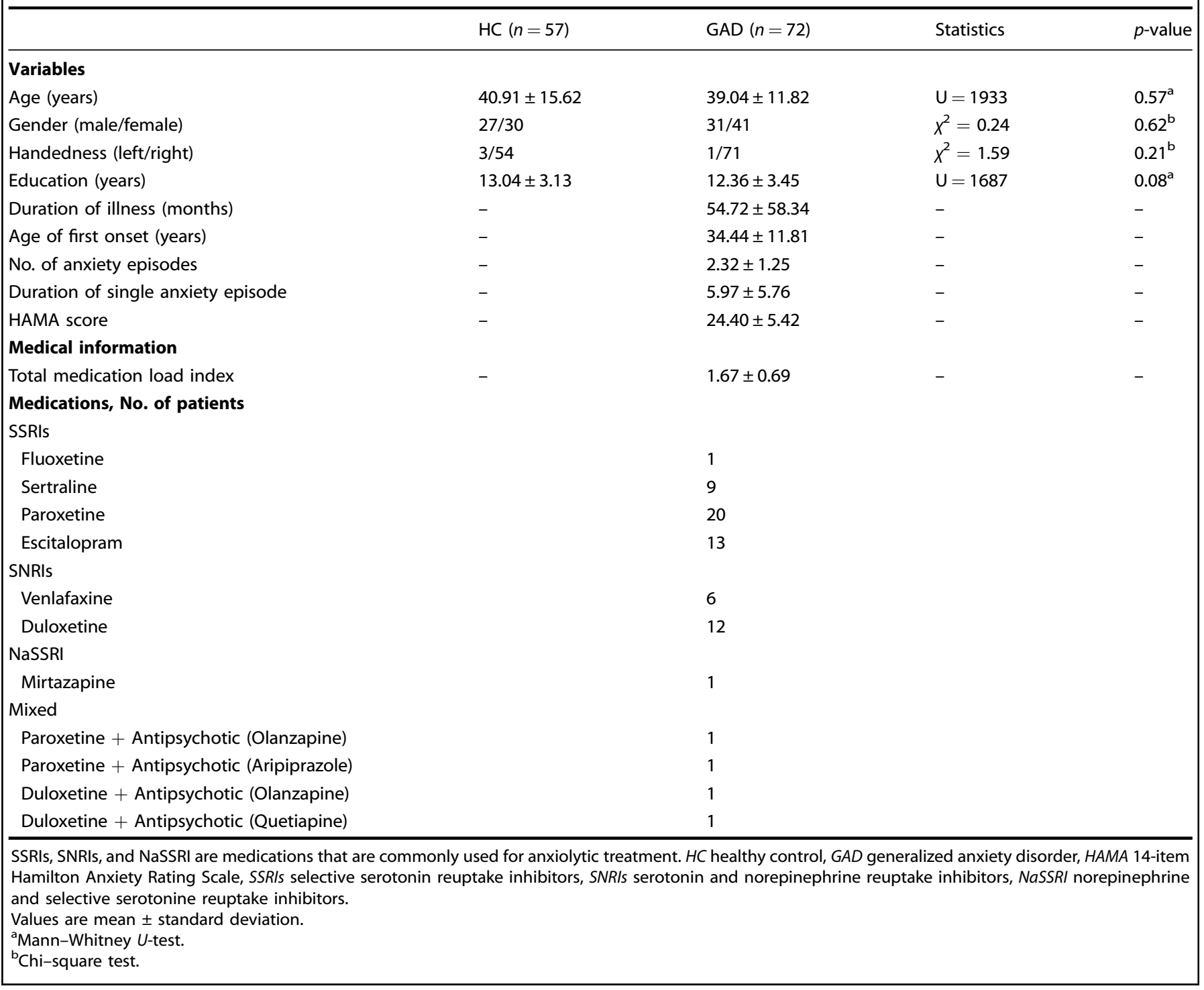

addition of HAMA scores as a new regressor. The Spearman correlation was performed between the GMV of each ROI and the illness duration of GAD, and the aforementioned variables except illness duration were controlled. The Bonferroni correction $(p<$ $0.05 / N$, where $N$ is the number of regions with a significant group difference in overall GMV) was used as the statistically significant level for the correlation analyses.

Subsequently, to explore the effect of gender on the GMV, we conducted the two-way analysis of variance (ANOVA) concerning two factors (i.e., diagnosis [GAD and $\mathrm{HC}$ ] and gender [male and female]) by using the full factorial model in SPM8. The demographic and the clinical characteristics of subjects who recruited into the gender-related analyses were presented in the Supplementary Table S3, and the unmatched variable between males and females with GAD (i.e., duration of single anxiety episode) was further considered in our imaging-related analyses. The detailed calculation steps of the two-way ANOVA analysis are presented in the Supplementary Materials.

Stage-specific GMV alteration patterns in patients with GAD The stage-specific GMV alterations were analyzed to simulate the progressive changes in the GMV of patients with GAD. We used two grouping strategies to divide the patients and explored and verified the changing patterns. The demographic and the clinical characteristics of the GAD subgroup of each grouping strategy were presented in the Supplementary Materials. The unmatched variables were further considered during the imaging-related analyses. In detail, given the distribution of illness duration and corresponding number of patients, the patients were divided arbitrarily into two stages (Stage 1: $0-3$ years; Stage 2: 4-20 years) and used as the main grouping strategy. The three years is the median of the illness duration of GAD, which can statistically divide the patients into two equal subgroups [40] and may benefit the match among the patient subgroups [29]. Moreover, the local polynomial regression fitting approach was used to observe the alteration tendency of the average GMV of the whole brain and the regions with significant overall GMV changes in GAD as the illness duration prolonged. As shown in the curves in Fig. 1b, c, the time points for early GMV fluctuation in GAD ranged from 25 to 50 months (nearly 2-4 years), which was close to the median duration. After comprehensively considering the median duration and the alteration trend of GMV, we chose three years to separate the patients with GAD. Subsequently, the GMV images of each subgroup of patients were compared with those of $\mathrm{HC}$ by using a 
A

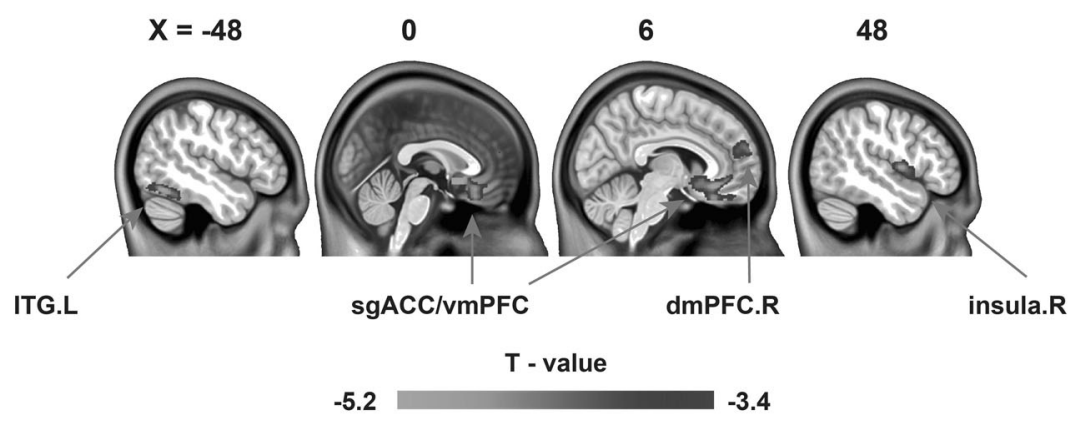

B average GMV of the whole brain C average GMV of regions with overall GMV alterations
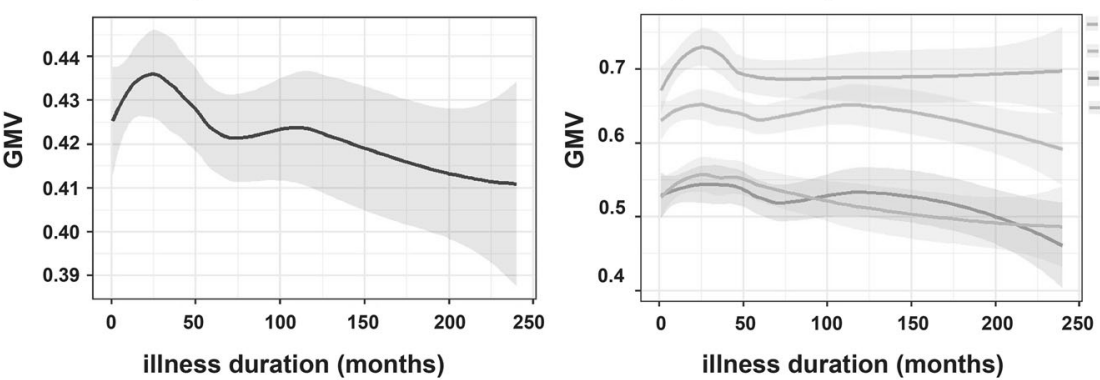
TTG.L SgACC/vmPFC dmPFC.R insula.R

Fig. 1 Overall GMV alterations and illness duration-related GMV alteration tendency of GAD. Overall GMV reduction in patients with GAD (GRF correction; voxel level $p<0.001$, cluster level $p<0.05$ ) (a). Alteration tendency of the average GMV of the whole brain of GAD (b) and GMV alteration tendency of regions with significant group differences in the overall GMV of GAD (c). GMV gray matter volume, GAD generalized anxiety disorder, ITG inferior temporal gyrus, sgACC subgenual anterior cingulate cortex, vmPFC ventromedial prefrontal cortex, dmPFC dorsal medial prefrontal cortex, L left, R right.

two-sample $t$-test (GRF correction; voxel level $p<0.001$, cluster level $p<0.05$ ). The age, gender, years of education, and TIV of subjects were used as covariates.

To verify the tendency of morphological alterations associated with illness duration, we further divided the patients into three stages (Stage 1: 0-2 years; Stage 2: 3-5 years; Stage 3: 6-20 years) and reanalyzed the data to reduce the influence of our group classification strategy on the results. We selected this validation grouping strategy because the number of patients in each stage was similar and may benefit the match among the patient subgroups. Moreover, the time points for the second GMV fluctuation of GAD were distributed from 40 to 75 months (nearly 3-6 years), which was approximately the span of the Stage 2 (Fig. 1b, c).

Additionally, we assessed the effects of gender, illness duration, and gender-by-duration interaction within the patient group by using a general linear model to examine the gender effect on the GMV as the illness duration increased. The detailed calculation process is presented in the Supplementary Materials.

Correlation between medications and GMV-related changes

To assess the possible influences of the medications on the GMVrelated findings, we conducted the Spearman correlation analysis of the total medication load index and the GMV of each ROI with significant between-group differences in the overall and the stage-specific GMV changes. The gender, age, years of education, TIV, illness duration, HAMA scores, illness onset, number of anxiety episodes, and the duration of single anxiety episode of patients were used as covariates. The threshold of $p<0.05 / N$ (Bonferroni correction) was employed as the statistically significant level for the correlation analysis. For the overall GMV changes, $N$ is the number of regions with significant group differences. For the stage-specific GMV changes, $N$ is the number of regions with significant between-group differences in each stage.
Seed-based CaSCN analysis for mapping the causal effects of the GMV alterations in one region on the whole brain GMV changes in GAD

The signed-path coefficient GC analysis was conducted to evaluate the time-lagged effects between brain regions and identify the directionality of influence. In past functional MRIrelated $\mathrm{GC}$ analyses, if the combination of the past time courses $X$ and $Y$ can better predict the current time course $Y$ than the past $Y$ alone, then the $X$ have a causal effect on $Y$ [41]. Similarly, when the GC method was applied to GMV data, the causal effects of GMV alterations can be observed. The positive GC value of region $A$ and negative $G C$ value of region $B$ indicated same and opposite GMV alterations, respectively, that occurred behind the seed region [26]. With GC analysis being time dependent, ranking the structural images of patients according to their illness duration from low to high before starting GC analysis was essential $[23,26]$. In this scenario, the "time" property can be assigned to structural images to make them suitable for GC analysis.

During the CaSCN computation, the seed regions were derived from the aforementioned GMV comparisons. Specifically, the subgenual ACC (sgACC) and the right insula were selected as seed regions because the former was considered the origin of GMV reduction in GAD. Moreover, the right insula showed abnormal GMV reduction as the illness duration increased and may be critical for the illness maintenance of GAD. Each seed was defined as an $8 \mathrm{~mm}$ radius sphere, and the mean values of the GMV of each seed were extracted from the sequenced data and constituted a pseudo-time series. The voxel-wise signed-path coefficient GC analysis was conducted on this series, and a CaSCN was constructed with the gender, age, years of education, TIV, illness onset, HAMA scores, total medication load index, number of anxiety episodes, and duration of single anxiety episode of 
patients as covariates. The calculation process was performed using the REST software (http://www.restfmri.net) [42]. For both seed regions, we only focused on the $G C$ value of $X$ to $Y$ given the presumption that the progressive GMV alterations of GAD were derived from the sgACC and the interests in which regions were influenced by the right insula during long illness duration. The GC map of each seed region was further transformed into a $Z$ score and corrected using the GRF method (voxel level $p<0.001$, cluster level $p<0.05$ ).

We additionally conducted the CaSCN analysis on the GMV data ranked using the patients' HAMA scores to examine the independent influences of the symptom severity on the causality of morphological alterations because some studies have demonstrated the correlations between the symptom severity and the GMV alterations in GAD [15, 17, 43, 44]. Moreover, we performed conjunction analyses between the CaSCN results using the data ranked by patients' illness duration and the symptom severity to investigate their common influences. Furthermore, we performed the sgACC- and the right insula-based CaSCN analyses in each gender and evaluated their group differences to map the gender-specific GMV changing patterns as the illness duration increased. These calculations are shown in the Supplementary Materials.

\section{RESULTS}

Overall GMV alterations in patients with GAD

The group differences of the overall GMV showed that patients with GAD exhibited decreased GMV compared with HC. The regions with decreased GMV were located in the left inferior temporal gyrus (ITG), bilaterial sgACC extending to the bilateral ventromedial prefrontal cortex (vmPFC), right dorsomedial prefrontal cortex (dmPFC), and right insula (GRF correction; voxel level $p<0.001$, cluster level $p<0.05$; Fig. $1 \mathrm{a}$ and Table 2). The alteration tendency of the average GMV of the whole brain and these four regions as the illness duration of GAD increased are presented in curves of Fig. 1b, c and used as the bases of grouping strategy in the subsequent stage-specific GMV analyses. Additionally, the GMV changes in each ROI were not significantly correlated with the HAMA scores, illness duration, and illness onset of GAD.

During the two-way ANOVA computation, the main effect of diagnosis replicated the overall GMV findings. The detailed results of the main effect of diagnosis and other effects are described in the Supplementary Materials and shown in Supplementary Fig. S1 and Table S4.

\begin{tabular}{|c|c|c|c|c|c|c|}
\hline \multirow[t]{2}{*}{ Brain regions } & \multirow{2}{*}{$\begin{array}{l}\text { Sphere } \\
\text { L/R }\end{array}$} & \multirow{2}{*}{$\begin{array}{l}\text { Cluster size } \\
\text { (voxels) }\end{array}$} & \multirow{2}{*}{$t$ values } & \multicolumn{3}{|c|}{ MNI Coordinates } \\
\hline & & & & $x$ & $y$ & z \\
\hline \multicolumn{7}{|l|}{ GAD $<$ HC } \\
\hline $\begin{array}{l}\text { sgACC extending } \\
\text { to vmPFC }\end{array}$ & $L / R$ & 3392 & -6.09 & 0 & 12 & -6 \\
\hline ITG & L & 689 & -5.21 & -48 & -60 & -18 \\
\hline Insula & $\mathrm{R}$ & 671 & -5.21 & 46.5 & 0 & 4.5 \\
\hline dmPFC & $\mathrm{R}$ & 408 & -4.39 & 6 & 61.5 & 21 \\
\hline
\end{tabular}

Stage-specific GMV alterations in patients with GAD

To investigate whether the reduction of GMV in patients with GAD had stage-specific characteristics and gradual changing patterns, we divided the patients into different stages according to their illness duration. Totally, GAD subgroups in different grouping strategies showed differences in terms of years of education, number of anxiety episodes, duration of single anxiety episode, and HAMA scores (Supplementary Tables S1 and S2), which were considered during the neuroimaging-related analyses. For the main grouping strategy, the GMV in patients with GAD gradually reduced with longer duration of illness. At Stage $1(n=37,14$ men; $36.27 \pm 11.28$ years; age range, $18-55$ years), only the sgACC exhibited reduced GMV in GAD. Aside from the sgACC, the bilateral vmPFC, left ITG, and right insula were observed with GMV reduction at Stage $2(n=35,17$ men; $41.97 \pm 11.82$ years; age range, 23-69 years). The GMV reduction originated from the sgACC and expanded to the frontal cortex, left temporal gyrus and right insula (Fig. 2a). Different grouping strategies of patients were implemented to reduce the bias of findings, which indicated a relevant tendency of GMV changes as the illness duration increased. these changes included the start point of GMV reduction and the critical regions for brain structural alterations during long illness duration. The sgACC remained the starting point for the progressive reduction of GMV in GAD, and the right insula emerged in long illness duration (Fig. 2b). The stage-specific GMV alterations were not significantly correlated with the HAMA scores, illness duration, and illness onset of GAD.

For the gender effect on the GMV as the illness duration of GAD increased, the specific results of the main effects of the gender and illness duration, as well as the effect of gender-by-duration interaction are reported in the Supplementary Materials and shown in Supplementary Fig. S2 and Table S5.

Effects of medications on GMV-related analysis

The overall and the stage-specific GMV changes were not significantly correlated with the total medication load index of $\mathrm{GAD}$, implicating that the medication had no significant influence on the morphological changes identified in this study.

Causal effects of GMV reduction in the sgACC and the right insula on the whole brain GMV alterations in GAD

The CaSCN analysis was performed by applying the GC analysis to the morphometric data that sequenced illness duration from low to high. The positive and the negative GC values of seedbased CaSCN analysis respectively represented same and opposite GMV changes in the regions that occurred behind the seed region [26]. Specifically, the GMV of the sgACC decreased in GAD. Therefore, the regions with a positive GC value in the sgACC-based CaSCN analysis had decreased GMV after the GMV reduction in sgACC. Conversely, the regions with a negative $G C$ value had increased GMV after the GMV reduction in the sgACC. Overall, during the sgACC- and the right insulabased $\mathrm{CaSCN}$ analyses, the regions involved in the parietal cortex exhibited consistent positive GC values, and the DLPFC, PCC, and parts of the temporal lobe and visual cortex showed consistent negative GC values. The ventrolateral prefrontal cortex (VLPFC) and the regions of the somatosensory cortex showed opposite GC values. Each seed region also exerted causal effects on some specific regions, which cannot be detected by another seed. Apart from the aforementioned regions, the sgACC had a positive causal influence on the medial prefrontal cortex (mPFC), right insula, and striatum (Fig. 3a and Supplementary Table S10). The right insula had a positive causal influence on the ACC and the supplementary motor area (SMA) and negative causal effects on the thalamus (Fig. $3 \mathrm{~b}$ and Supplementary Table S11). The summary of the sgACC- and the right insula-based $\mathrm{CaSCN}$ analyses is presented in Fig. $3 \mathrm{C}$. 
A
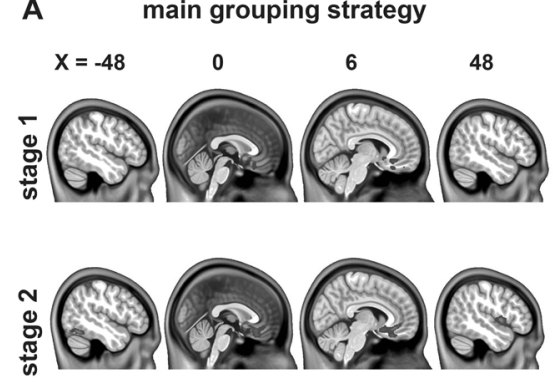

T - value
B

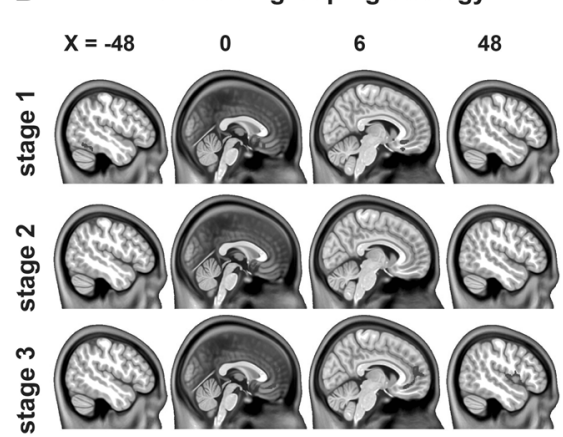

Fig. 2 Stage-specific GMV changes in GAD patients under different grouping strategies. All group differences were corrected using the GRF method (voxel level $p<0.001$, cluster level $p<0.05$ ). The stages in the main grouping strategy were categorized using the illness duration of GAD (stage 1, 0-3 years; stage 2: 4-20 years) (a). The stages in the validation grouping strategy were separated using the illness duration of GAD (stage 1, 0-2 years; stage 2, 3-5 years; stage 3, 6-20 years) (b). GMV gray matter volume, GAD generalized anxiety disorder.

The seed-based CaSCN analysis of the data ranked using the patients' symptom severity showed similar regions with those of the data ranked using the illness duration of GAD, whereas the causality was not exactly the same (Supplementary Fig. S3, Supplementary Tables S12 and S13). Some regions showed consistent causality with the given seeds with increased illness duration and aggravation in anxiety symptom during the conjunction analyses (Supplementary Fig. S4, Supplementary Tables S6 and S7). Intriguingly, the causality of the brain morphological alterations were not exactly the same in male and female patients, which may suggest the different patterns in the brain structural alterations in males and females with GAD as the illness duration prolonged (Supplementary Figs. S5 and S6, Supplementary Tables S8 and S9). The detailed results are described in the Supplementary Materials.

\section{DISCUSSION}

We have investigated the causal relationships of morphometric alterations in GAD as the illness duration is prolonged by applying the GC analysis to high-resolution T1-weighted structural images. The GMV in the sgACC decreased in the early stage of the disease and gradually expands to the bilateral vmPFC, right dmPFC, left ITG, and the right insula as the illness duration increases. The gradual GMV reduction tendency in patients with GAD is found in stage-specific morphological comparisons and confirmed using the sgACC-based CaSCN analysis. A small GMV in the right insula is identified in long illness duration and may be important for the maintenance of anxiety given the essential role of the insula in anxiety disorders. The right insula has a positive causal effect on numerous areas involving the sgACC. The bidirectional positive causal effects between the sgACC and the right insula may aggravate each other's GMV reduction. Additionally, the supplemental analyses suggest that the causality between some regions may jointly influenced by the illness duration and the symptom severity of GAD, and the structural alteration patterns in males and females with GAD may not the same as the illness duration increases. Although the supplemental results are not used as the main findings of this study, these findings provide comprehensive information for observing and understanding the brain structural alterations in GAD.

During the overall GMV comparison, some of our findings are consistent and some are divergent with those of previous morphometric studies. For instance, our finding on the reduced GMV in the insula cortex concurs with previous morphologicalbased findings regarding $\operatorname{GAD}[17,18]$. The insula is a pivotal region for interoceptive emotional awareness and responses [45] and considered a critical region for understanding anxiety proneness [46]. In patients with GAD, GMV reduction is also observed in the sgACC, which belongs to the ventral ACC [47] and exerts bidirectional anatomical connection and strong functional coupling with the insula [48]. The structural impairments in the insula and the ACC may disrupt their functional coupling and may be associated with the abnormal interoceptive processing of GAD [49]. The vmPFC and the ITG have also exhibited decreased GMV in GAD. These two regions are involved in the DMN, which is essential for self-referential processing and emotion regulation $[50,51]$. The inappropriate self-referential thinking $[52,53]$ and failure of emotion regulation of GAD $[54,55]$ may be explained partly by the structural alteration of the DMN and its abnormal coordination with other networks since the disrupted coordination may be essential for the pathology of several psychiatric and neurological disorders $[56,57]$. Notably, a study has identified increased GMV in the dmPFC of nonmedicated adult females with GAD [15]. Nevertheless, the present work demonstrated a decreased GMV of dmPFC in GAD. This discrepancy may be due to the different subtypes of patients, medication treatment, and sample size. Additional neuroimaging studies may be necessary to verify this result.

The stage-specific morphological analysis of different grouping strategies shows that the GMV reduction in the sgACC and the right insula are found respectively in the early stage and in longer illness duration of GAD, an outcome that may be important for understanding the dynamic morphometric alterations of GAD along with increasing illness duration. The sgACC-based CaSCN analysis can assess the causal influence of the GMV reduction in the sgACC on the gradual structural changes in GAD, and the right insula-based CaSCN analysis can identify how the morphological abnormality in longer illness duration reversely influences the brain structures of other regions. Interestingly, the sgACC and the right insula project a reciprocal positive causal effect on each other. Considering that a positive GC value may imply the same GMV alteration with the seed region [26], the GMV reduction in the right insula may lag behind the sgACC and, in turn, aggravate the GMV reduction in the sgACC. The insula is also responsible for salient stimuli detection except for the interoceptive processing and the ACC is prominent in generating appropriate control signals by cooperating with the insula [48]. Menon and colleagues have postulated that the anterior insula is overactive in anxiety disorders when responding to salient stimuli, and the increased anxiety may be caused by the misattributed emotional salience of the anterior insula [48]. The insula activation occurs in response to worry sentences [58] and ambiguous affective stimuli [59] in GAD. Enhanced insula activity may serve as a compensatory response for its structural alteration but may be increased excessively. The structural deficits of the insula and the ACC may be associated with the abnormal salience processing and disintegration of the bottom-up attention switching and the top-down attentional control in GAD. 
A Seed: sgAcc
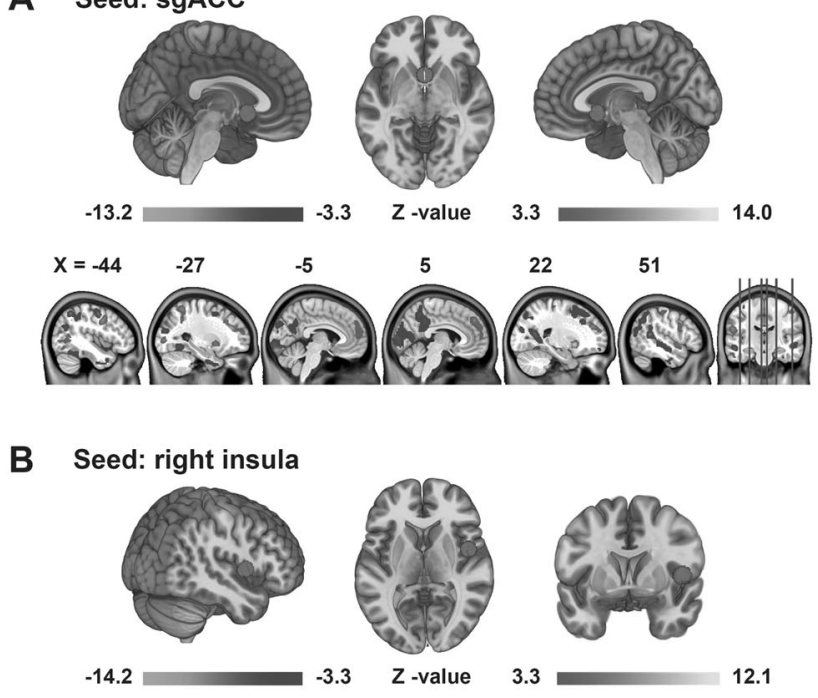

Z -value
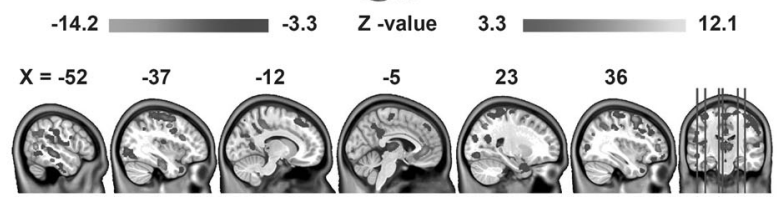

Fig. 3 Seed-based CaSCN results. Regions with causal effects on the gray matter reduction in the sgACC (a) and the right insula (b) in patients with GAD (GRF correction; voxel level $p<0.001$, cluster level $p<0.05$ ). Summary of the results of Graphs $A$ and B (c). The arrow lines in Graph C represent GC value. The bold arrow lines indicate positive or negative causal effects from the sgACC and the right insula. The thin arrow lines represent the positive or negative causal effects from the sgACC or the right insula alone. CaSCN causal structural covariance network, sgACC subgenual anterior cingulate cortex, GAD generalized anxiety disorder, GC Granger causality.

During the CaSCN analysis, the sgACC and the right insula may jointly facilitate the GMV reduction in the parietal cortex, which is consistent with a previous finding that the GMV in the parietal cortex of GAD decreases [13] and may be influenced by the GMV reduction in the sgACC and the right insula. The interpretation is based on the assumption that the influence of the decreased GMV in the sgACC occurs at the early stage, whereas the effects of insula happens with long illness duration, a notion that also applies in the following explanations. The parietal cortex is anchored in the frontoparietal control network [60], which is essential for cognitive reappraisal in anxiety disorders [61]. Patients with anxiety disorders have been reported to show decreased activation in the parietal cortex during cognitive reappraisal [62]. The structural alterations of the parietal cortex may be parallel with such functional abnormality and provide a neurostructural basis for this neurofunctional phenomena [13]. Moreover, the PCC, DLPFC, and most subregions of the temporal lobe and visual cortex have a negative GC value, which may be interpreted as a compensatory effect responsible for the GMV reduction in the sgACC and the right insula as the illness duration is prolonged. The sgACC and the right insula also exert opposite causal effects on the somatosensory cortex and VLPFC, potentially leading to different orientations of GMV changes in these cortices. The opposite causal effects may provide a new perspective for understanding the opposite findings of the GMV changes in the somatosensory cortex $[12,16]$. The deficient top-down control of the VLPFC on the subcortical regions (i.e, amygdala) [63], a neural basis of emotion dysregulation in GAD [55], may correlated with the abnormality of the VLPFC.

Except the abovementioned regions simultaneously affected by sgACC and right insula, the MPFC and the striatum may also be observed with sgACC-induced GMV reduction. The decreased GMV in the right insula may subsequently lead to GMV reduction in the SMA and GMV enlargement in the thalamus. Large GMVs in the striatum [21, 43] and the dmPFC [15] are found in GAD and positively correlate with the worry severity of patients [43]. The putative GMV reduction in the striatum and the dmPFC may be interpreted as a compensatory effect of the GMV reduction in the sgACC. Hilbert and colleagues have reported that the GMV in SMA is negatively correlated with the worry severity of GAD [43]. Our findings on the GMV reduction in the SMA may indicate that the anxiety severity of GAD may be exacerbated. The increased GMV in the thalamus is in line with the finding of a prior study on pediatric GAD [21]. The thalamus is a major relay center that has strong reciprocal connections with the cortical and the subcortical regions to filter sensory information and engage in high cognitive functions and emotional regulation [64, 65]. The increased GMV in the thalamus may be partially associated with the abnormal sensory information and the high-order cognitive and emotion processing of GAD.

This study has several limitations that must be considered. First, the stage-specific morphological analyses are confined by the number of patients and the distribution of their illness duration, and the arbitrary grouping strategies may affect the association between the GMV alterations and the illness duration. Second, the CaSCN analysis is used to examine the chronological order of GMV changes by sequencing the GMV data in accordance with the illness duration of GAD. This approach cannot directly reflect the real temporal sequence of brain structural alterations. Third, we cannot confidently determine whether the causality of the structural changes observed in this study are specific to illness duration because the causality between some regions are simultaneously correlated with the illness duration and the anxiety symptom of GAD. In this scenario, the CaSCN results based on illness duration should be interpreted cautiously and validated through longitudinal studies in the future. Fourth, when patients are divided into different genders, the relatively small sample size in each subgroup may affect the statistical power, and the gender-related findings are expected to be confirmed by larger samples. Fifth, considering that previous findings have demonstrated the significant effects of medications on the brain function and the structure of GAD [66-68] and that no 
medication-free patient control group is involved in this study, we cannot exclude the potential effects of medications although no significant correlation is observed. We expect that future studies with medication-free samples can replicate our results. Finally, the years of education is not adequately matched between patients and $\mathrm{HC}$ during the stage-specific GMV comparisons. Although this indicator is used as a covariate in neuroimaging analyses, its confounding effect on morphological changes should be further considered.

\section{CONCLUSION}

The stage-specific group comparison of GMV and seed-based CaSCN analysis were used to simulate the temporal information and characterize the causal relationships of the brain structural changes in patients with GAD as the illness duration increased. The patients with GAD exhibited gradual GMV reduction, in which the sgACC was the origin of the morphological changes and the right insula may be important for illness maintenance. In addition to the reciprocal causal effects between the sgACC and the right insula, the sgACC and the right insula exerted causal effects on regions involved in the frontal, parietal, visual cortices, temporal lobe, and the somatosensory cortex. These findings may facilitate our understanding on the structural alterations in GAD as the illness duration increased and may provide (pre)clinical implications for anxiety intervention and treatment by identifying the critical regions with structural alterations in GAD. Additionally, the supplemental symptom severity and gender-related findings uncovered potential influences of these factors on brain structural alterations in $G A D$, which remind us to be cautious in understanding our main findings and provide some clues for future morphologicalrelated studies of GAD.

\section{FUNDING AND DISCLOSURE}

This study was supported by the Key Project of Research and Development of Ministry of Science and Technology (2018AAA0100705), the Natural Science Foundation of China (61533006, U1808204, 81771919), the Scientific research project of Sichuan Medical Association (S15012), the Youth Innovation Project of Sichuan Provincial Medical Association (Q14014), the Fundamental Research Funds for the Central Universities (ZYGX2013Z004), Sichuan Science and Technology Program (2018TJPT0016), and the China Postdoctoral Science Foundation Grant (2019M653383). All authors declare that they have no conflicts of interest.

\section{ACKNOWLEDGEMENTS}

The authors thank all subjects who participate in this study.

\section{AUTHOR CONTRIBUTIONS}

Yuyan Chen, Qian Cui, and Huafu Chen designed the study. Yuyan Chen, Qin Tang, Di Li, Ting Lei, Wei Sheng, Yang Yang, Shan Hu, and Jiaxin Deng collected and analyzed the data. Yuyan Chen and Qian Cui drafted the work. Yunshuang Fan, Xiaonan Guo, Fengmei Lu, Zongling $\mathrm{He}$, and Huafu Chen revised the draft.

\section{REFERENCES}

1. Tyrer P, Baldwin D. Generalised anxiety disorder. Lancet 2006;368:2156-66.

2. Fonzo GA, Etkin A. Brain connectivity reflects mental and physical states in generalized anxiety disorder. Biol Psychiatry 2016;80:733-5.

3. Makovac E, Meeten F, Watson DR, Herman A, Garfinkel SN, Critchley HD, et al. Alterations in amygdala-prefrontal functional connectivity account for excessive worry and autonomic dysregulation in generalized anxiety disorder. Biol Psychiatry 2016;80:786-95.
4. Cuijpers P, Sijbrandij M, Koole S, Huibers M, Berking M, Andersson G. Psychological treatment of generalized anxiety disorder: a meta-analysis. Clin Psychol Rev. 2014;34:130-40.

5. Makovac E, Fagioli S, Watson DR, Meeten F, Smallwood J, Critchley HD, et al. Response time as a proxy of ongoing mental state: a combined $\mathrm{fMRI}$ and pupillometry study in Generalized Anxiety Disorder. Neuroimage 2019;191:380-91.

6. DeMartini J, Patel G, Fancher TL. Generalized anxiety disorder. Annal. Internal Med. 2019;170:ITC49-64.

7. Kolesar TA, Bilevicius E, Wilson AD, Kornelsen J. Systematic review and metaanalyses of neural structural and functional differences in generalized anxiety disorder and healthy controls using magnetic resonance imaging. NeuroimageClin. 2019;24:102016.

8. Rutter LA, Scheuer L, Vahia IV, Forester BP, Smoller JW, Germine L. Emotion sensitivity and self-reported symptoms of generalized anxiety disorder across the lifespan: a population-based sample approach. Brain Behav. 2019;9:e01282

9. Denefrio S, Myruski S, Mennin D, Dennis-Tiwary TA. When neutral is not neutral: neurophysiological evidence for reduced discrimination between aversive and non-aversive information in generalized anxiety disorder. Motiv Emot. 2019;43:325-38.

10. Moon C-M, Jeong G-W. Brain morphological alterations and cellular metabolic changes in patients with generalized anxiety disorder: a combined DARTELbased VBM and (1) H-MRS study. Magn Reson Imaging. 2016;34:429-36.

11. Hilbert K, Lueken U, Beesdo-Baum K. Neural structures, functioning and connectivity in generalized anxiety disorder and interaction with neuroendocrine systems: a systematic review. J Affect Disord. 2014;158:114-26.

12. Strawn JR, Wehry AM, Chu W-J, Adler CM, Eliassen JC, Cerullo MA, et al. Neuroanatomic abnormalities in adolescents with generalized anxiety disorder: a voxel-based morphometry study. Depression Anxiety. 2013;30:842-8.

13. Strawn JR, Hamm L, Fitzgerald DA, Fitzgerald KD, Monk CS, Phan KL. Neurostructural abnormalities in pediatric anxiety disorders. J Anxiety Disord. 2015;32:81-8.

14. Shang J, Fu Y, Ren Z, Zhang T, Du M, Gong Q, et al. The common traits of the ACC and PFC in anxiety disorders in the DSM-5: meta-analysis of voxel-based morphometry studies. Plos One 2014;9:e93432.

15. Schienle A, Ebner F, Schaefer A. Localized gray matter volume abnormalities in generalized anxiety disorder. Eur Arch Psychiatry Clin Neurosci. 2011;261:303-7.

16. Makova E, Meeten F, Watson DR, Garfinkel SN, Critchley HD, Ottaviani C. Neurostructural abnormalities associated with axes of emotion dysregulation in generalized anxiety. Neuroimage-Clin. 2016;10:172-81.

17. Moon C-M, Kim G-W, Jeong G-W. Whole-brain gray matter volume abnormalities in patients with generalized anxiety disorder: voxel- based morphometry. Neuroreport 2014;25:184-9.

18. Moon C-M, Yang J-C, Jeong G-W. Explicit verbal memory impairments associated with brain functional deficits and morphological alterations in patients with generalized anxiety disorder. J Affect Disord. 2015;186:328-36.

19. De Bellis MD, Keshavan MS, Shifflett H, lyengar S, Dahl RE, Axelson DA, et al. Superior temporal gyrus volumes in pediatric generalized anxiety disorder. Biol Psychiatry 2002;51:553-62.

20. De Bellis MD, Casey BJ, Dahl RE, Birmaher B, Williamson DE, Thomas KM, et al. A pilot study of amygdala volumes in pediatric generalized anxiety disorder. Biol Psychiatry 2000;48:51-7.

21. Liao $M$, Yang $F$, Zhang $Y$, He Z, Song $M$, Jiang $T$, et al. Childhood maltreatment is associated with larger left thalamic gray matter volume in adolescents with generalized anxiety disorder. Plos One 2013;8:e71898.

22. Liao M, Yang F, Zhang Y, He Z, Su L, Li L. Lack of gender effects on gray matter volumes in adolescent generalized anxiety disorder. J Affect Disord. 2014;155:278-82.

23. Zhang Z, Liao W, Xu Q, Wei W, Zhou HJ, Sun K, et al. Hippocampus-associated causal network of structural covariance measuring structural damage progression in temporal lobe epilepsy. Hum Brain Mapp. 2017;38:753-66.

24. Seth $A K$, Barrett $A B$, Barnett $L$. Granger causality analysis in neuroscience and neuroimaging. J Neurosci. 2015;35:3293-7.

25. Liao W, Fan Y-S, Yang S, Li J, Duan X, Cui Q, et al. Preservation effect: cigarette smoking acts on the dynamic of influences among unifying neuropsychiatric triple networks in schizophrenia. Schizophrenia Bull 2019:45:1242-50.

26. Jiang $Y$, Luo $C$, Li X, Duan $M, H e ~ H, C h e n ~ X$, et al. Progressive reduction in gray matter in patients with schizophrenia assessed with MR imaging by using causal network analysis. Radiology 2018;287:633-42.

27. Moran LV, Tagamets MA, Sampath H, O'Donnell A, Stein EA, Kochunov P, et al. Disruption of anterior insula modulation of large-scale brain networks in schizophrenia. Biol Psychiatry 2013;74:467-74.

28. Wang J, Wei Q, Bai T, Zhou X, Sun H, Becker B, et al. Electroconvulsive therapy selectively enhanced feedforward connectivity from fusiform face area to 
amygdala in major depressive disorder. Soc Cogn Affect Neurosci. 2017;12:1983-92.

29. Li Y, Wang C, Teng C, Jiao K, Song X, Tan Y, et al. Hippocampus-driving progressive structural alterations in medication-naive major depressive disorder. J Affect Disord. 2019;256:148-55.

30. Gale C, Davidson O. Generalised anxiety disorder. Bmj-Br Med J. 2007;334:579-81.

31. Angst J, Gamma A, Baldwin DS, Ajdacic-Gross V, Roessler W. The generalized anxiety spectrum: prevalence, onset, course and outcome. Eur Arch Psychiatry Clin Neurosci. 2009;259:37-45.

32. Steiner M, Allgulander C, Ravindran A, Kosar H, Burt T, Austin C. Gender differences in clinical presentation and response to sertraline treatment of generalized anxiety disorder. Hum Psychopharmacol-Clin Exp. 2005;20:3-13.

33. Cui Q, Chen Y, Tang Q, Han S, Hu S, Pang Y, et al. Disrupted dynamic local brain functional connectivity patterns in generalized anxiety disorder. Prog NeuroPsychopharmacol Biol Psychiatry. 2020;99:109833.

34. Cui Q, Sheng W, Chen Y, Pang Y, Lu F, Tang Q, et al. Dynamic changes of amplitude of low-frequency fluctuations in patients with generalized anxiety disorder. Hum Brain Mapp 2020;41:1667-76.

35. Chen Y, Cui Q, Xie A, Pang Y, Sheng W, Tang Q, et al. Abnormal dynamic functional connectivity density in patients with generalized anxiety disorder. J Affect Disord. 2020;261:49-57.

36. Hassel S, Almeida JRC, Kerr N, Nau S, ladouceur CD, Fissell K, et al. Elevated striatal and decreased dorsolateral prefrontal cortical activity in response to emotional stimuli in euthymic bipolar disorder: no associations with psychotropic medication load. Bipolar Disord 2008;10:916-27.

37. Versace A, Almeida JRC, Hassel S, Walsh ND, Novelli M, Klein CR, et al. Elevated left and reduced right orbitomedial prefrontal fractional anisotropy in adults with bipolar disorder revealed by tract-based spatial statistics. Arch Gen Psychiatry. 2008;65:1041-52.

38. Pang $Y$, Chen $H$, Wang $Y$, Long $Z$, He Z, Zhang $H$, et al. Transdiagnostic and diagnosis-specific dynamic functional connectivity anchored in the right anterior insula in major depressive disorder and bipolar depression. Prog NeuroPsychopharmacol Biol Psychiatry. 2018;85:7-15.

39. Sackeim HA. The definition and meaning of treatment-resistant depression. J Clin Psychiatry. 2001;62:10-7.

40. Walsh JE. Applications of some significance tests for the median which are valid under very general conditions. J Am Stat Assoc. 1949:44:342-55.

41. Granger CWJ. Investigating causal relations by econometric models and crossspectral methods. Econometrica 1969;37:424-38.

42. Zang Z-X, Yan C-G, Dong Z-Y, Huang J, Zang Y-F. Granger causality analysis implementation on MATLAB: a graphic user interface toolkit for fMRI data processing. J Neurosci Methods. 2012;203:418-26.

43. Hilbert K, Pine DS, Muehlhan M, Lueken U, Steudte-Schmiedgen S, Beesdo-Baum K. Gray and white matter volume abnormalities in generalized anxiety disorder by categorical and dimensional characterization. Psychiatry Res-Neuroimaging. 2015;234:314-20.

44. Ma Z, Wang C, Hines CS, Lu X, Wu Y, Xu H, et al. Frontoparietal network abnormalities of gray matter volume and functional connectivity in patients with generalized anxiety disorder. Psychiatry Res-Neuroimaging. 2019;286:24-30.

45. Craig AD. How do you feel-now? The anterior insula and human awareness. Nat Rev Neurosci. 2009;10:59-70.

46. Paulus MP, Stein MB. An insular view of anxiety. Biol Psychiatry 2006;60:383-7.

47. Lockwood PL, Wittmann MK. Ventral anterior cingulate cortex and social decision-making. Neurosci Biobehav Rev. 2018;92:187-91.

48. Menon V, Uddin LQ. Saliency, switching, attention and control: a network model of insula function. Brain Struct Funct. 2010;214:655-67.

49. Pang J, Tang $X$, Li H, Hu Q, Cui H, Zhang L, et al. Altered interoceptive processing in generalized anxiety disorder a heartbeat-evoked potential research. Front Psychiatry. 2019;10:616.
50. Sylvester CM, Corbetta M, Raichle ME, Rodebaugh TL, Schlaggar BL, Sheline $\mathrm{YI}$ et al. Functional network dysfunction in anxiety and anxiety disorders. Trends Neurosci. 2012;35:527-35.

51. Menon V. Large-scale brain networks and psychopathology: a unifying triple network model. Trends Cogn Sci. 2011;15:483-506.

52. Fresco DM, Roy AK, Adelsberg S, Seeley S, Garcia-Lesy E, Liston C, et al. Distinct functional connectivities predict clinical response with emotion regulation therapy. Frontiers in human. Neuroscience 2017;11:86.

53. Mennin DS, Fresco DM, O'Toole MS, Heimberg RG. A randomized controlled trial of emotion regulation therapy for generalized anxiety disorder with and without co-occurring depression. J Consulting Clin Psychol. 2018;86:268-81.

54. Palm ME, Elliott R, McKie S, Deakin JFW, Anderson IM. Attenuated responses to emotional expressions in women with generalized anxiety disorder. Psychological Med 2011;41:1009-18.

55. Mochcoyitch MD, da Rocha Freire RC, Garcia RF, Nardi AE. A systematic review of fMRI studies in generalized anxiety disorder: evaluating its neural and cognitive basis. J Affect Disord. 2014;167:336-42.

56. Guo X, Duan X, Suckling J, Chen H, Liao W, Cui Q, et al. Partially impaired functional connectivity states between right anterior insula and default mode network in autism spectrum disorder. Hum Brain Mapp. 2019;40:1264-75.

57. Wang J, Wei Q, Wang L, Zhang H, Bai T, Cheng L, et al. Functional reorganization of intra- and internetwork connectivity in major depressive disorder after electroconvulsive therapy. Hum Brain Mapp. 2018;39:1403-11.

58. Hoehn-Saric R, Schlund MW, Wong SHY. Effects of citalopram on worry and brain activation in patients with generalized anxiety disorder. Psychiatry ResNeuroimaging. 2004;131:11-21.

59. Simmons A, Matthews SC, Paulus MP, Stein MB. Intolerance of uncertainty correlates with insula activation during affective ambiguity. Neurosci Lett 2008;430:92-7.

60. Wang $H-Y$, Xu G-Q, Ni M-F, Zhang $C-H$, Sun X-P, Chang $Y$, et al. Neural mechanisms of implicit cognitive reappraisal: preceding descriptions alter emotional response to unpleasant images. Neuroscience 2017;347:65-75.

61. Blair KS, Geraci M, Smith BW, Hollon N, DeVido J, Otero M, et al. Reduced dorsal anterior cingulate cortical activity during emotional regulation and top-down attentional control in generalized social phobia, generalized anxiety disorder, and comorbid generalized social phobia/generalized anxiety disorder. Biol Psychiatry 2012;72:476-82.

62. Wang H-Y, Zhang X-X, Si C-P, Xu Y, Liu Q, Bian H-T, et al. Prefrontoparietal dysfunction during emotion regulation in anxiety disorder: a meta-analysis of functional magnetic resonance imaging studies. Neuropsychiatr Dis Treat 2018;14:1183-98.

63. Monk CS, Telzer EH, Mogg K, Bradley BP, Mai X, Louro HMC, et al. Amygdala and ventrolateral prefrontal cortex activation to masked angry faces in children and adolescents with generalized anxiety disorder. Arch Gen Psychiatry. 2008;65:568-76

64. Herrero MT, Barcia C, Navarro JM. Functional anatomy of thalamus and basal ganglia. Childs Nerv Syst. 2002;18:386-404.

65. Haber SN, Calzavara R. The cortico-basal ganglia integrative network: the role of the thalamus. Brain Res Bull. 2009;78:69-74.

66. Brown GG, Ostrowitzki S, Stein MB, von Kienlin M, Liu TT, Simmons A, et al. Temporal profile of brain response to alprazolam in patients with generalized anxiety disorder. Psychiatry Res-Neuroimaging. 2015;233:394-401.

67. Abdallah CG, Coplan JD, Jackowski A, Sato JR, Mao X, Shungu DC, et al. A pilot study of hippocampal volume and $\mathrm{N}$-acetylaspartate (NAA) as response biomarkers in riluzole-treated patients with GAD. Eur Neuropsychopharmacol 2013:23:276-84.

68. Abdallah CG, Coplan JD, Jackowski A, Sato JR, Mao X, Shungu DC, et al. Riluzole effect on occipital cortex: a structural and spectroscopy pilot study. Neurosci Lett 2012;530:103-7. 